\title{
Issues in Microbial Risk Assessment
}

\author{
M. Cohen ${ }^{1}$ T. H. Taylor, Jr. ${ }^{2}$ T. Whalen ${ }^{3}$ \\ ${ }^{1}$ Frontline Healthcare Workers Safety Foundation, Atlanta Georgia USA \\ ${ }^{2}$ Centers for Disease Control and Prevention, Atlanta, Georgia, USA (The findings and \\ conclusions in this report are those of the authors and do not necessarily represent the \\ official position of the Centers for Disease Control and Prevention) \\ ${ }^{3}$ Georgia State University, Atlanta Georgia USA; tom@whalen3.org
}

\begin{abstract}
Risk assessment is the qualitative or quantitative characterization of the potential health effects of particular substances on individuals or populations. Practical public health policy/decision making increasingly requires significant adjustments in microbial risk assessments, and the U.S. federal regulatory community anticipates greater conformity and consistency in microbial risk assessment. Alternative approaches include those based on possibility theory, agent-based explicit spatial-temporal simulation, and maximum possible risk analysis built on counterfactual assumptions.
\end{abstract}

Keywords: microbial risk assessment, modeling, acceptable risks, possibilistic, possibility theory, agent-based-explicit spatial-temporal simulation, worst-case, maximum possible risk

\section{Background}

The seminal book Risk Assessment in the Federal Government; Managing the Process, from the U.S. National Academies [1] defines risk assessment as "the qualitative or quantitative characterization of the potential health effects of particular substances on individuals or populations." $\quad$ Risk assessments include hazard identification, exposure assessment, dose-response analysis, and risk characterization. For a potentially harmful event, the classical definition of risk incorporates the probability of the event and how great the impact (loss or cost) of that event would be if it occurred. Mathematically, the risk of a potentially harmful event is determined by the possible adverse impacts, and the probability of each one occurring. [2]

Classical infectious disease risk modeling has focused either on identifying effects of various public health controls or intervention elements, such as with influenza or salmonellosis; or on achieving zero-cases, such as with vaccinations for smallpox or polio. So long as success is measured by zero, then risk is neither being assessed nor managed in a practical sense. Public health policy/decision-making requires acceptance of trade-offs and some determination of acceptable risks. Since resources are finite, whenever one risk is set at zero, then it necessarily requires an increase in the risk of something else. An example of this would be a determination of the impact on public health by treating drinking water with a chemical (risk to chlorine exposure) versus the impact of exposure to pathogenic organisms in water that is not treated. [3]

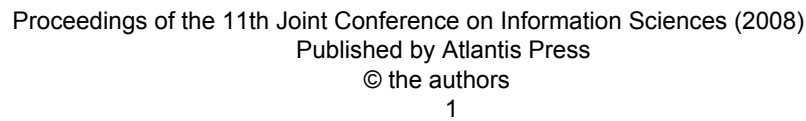


As new information on the identification of infectious microbial pathogens, their occurrence, potential for human exposure, dose response, and attributable health effects has become increasingly available over the past decade, the need for significant adjustments in microbial risk assessments has gained momentum in the U.S. federal regulatory community. [3]

Classical probability and risk analysis is appropriate for well-characterized, probable events. However, many real world events - and certainly those pertaining to current issues in microbial risk assessment - include adventitious components whose probabilities are not measurably different from zero. For those, classical risk analysis breaks down and alternative approaches are required. [4]

Since the anthrax attacks of 2001, there has been growing awareness of the potential for the deliberate use by malicious persons of biological agents that pose a 'credible' threat to the health and welfare of the exposed population and the economy. It may be necessary to assess the risks associated with deliberate introduction of harmful biological agents into the water supply, food supply, or release into highly populated indoor and outdoor public areas as an aerosol. Information such as projected exposure and attendant health impacts should be factored in to determine the probability of occurrence and associated consequences of a particular event. However, there is no well defied formula to quantify the risks associated with the intentional release of a biological agent. [3]

A number of credible mathematical models, protocols, and other tools have become available that allow preparation of quantitative risk assessments in microbiology to be conducted even where there is a great deal of variability, uncertainty, and lack of specific data. [3] The complex interplay between pathogen-host-environment has required that models and simulations address specific aspects of microbiological agents including strain differentiation, detection difficulty in the environment, various exposure scenarios, variable host susceptibilities in a susceptible population, a spectrum of health effects, and variable presentation of symptoms. [5]

Possibilistic risk management focuses on reducing the relevance of historical events by countermeasures to prevent their recurrence. Possibility theory separates the probability and possibility of disease after a release of hazardous material from the probability and possibility of that release. [6] The confidence interval and point estimate of disease probability can be calculated using appropriate toxicological and microbiological techniques. [7]

In a companion paper [8], possibility theory and agent-based explicit spatialtemporal simulation are used to compare the effects on each of three real communities given the assumption that a rare disease is carried out of a hypothetical high containment biological research laboratory sited in each community. The initial event has nonzero possibility, but its probability is not well measureably different from zero. The conditional distributions obtained by making this event an input to the simulation resemble "counterfactual conditionals" that can provide useful information about the relative technical and social desirability of alternative sites, even though a conventional risk assessment is not possible.

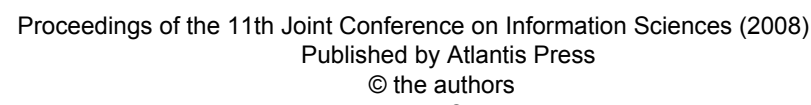


In another companion paper [9], counterfactual assumptions enable a maximum possible risk (MPR) analysis of the possibility of an aerosol release of pathogens such as anthrax spores from a biological research laboratory. It is possible to conclude that the laboratory is safe without attempting to measure its actual risk or specify an actual threshold of acceptable risk. The logic of this socalled MPR modeling is that if it is possible to prove that the model risk is greater than the actual risk without precisely quantifying the model risk, and that the model risk is less than the acceptable risk without precisely quantifying the acceptable risk, then we can demonstrate that the actual risk is less than the acceptable risk without precisely quantifying either.

The U.S. regulatory community anticipates increased coordination or at least conformity/consistency in microbiological risk assessments. Advanced methods and innovative approaches are needed to bridge gaps in characterizing microbes, gain a better understanding of susceptible populations, and to set priorities for research initiatives. [10]

A thorough evaluation of key sources of uncertainty and variability in risk characterizations can lead to more productive research efforts and a better use of limited resources in terms of both risk assessment and risk management. The historical approach used by many to present overly conservative "worst-case" estimates of risk based on limited data will no longer be considered acceptable, or necessarily in the best interests of society.[2]

\section{References}

[1] NRC (National Research Council). Risk Assessment in the Federal Government: Managing the Process. Washington, DC: National Academy Press 1983.

[2] Williams, RD; Paustenbach, D. Risk Characterization: Principles and Practice, J.of Toxicology \& Env Health: Part B/2002-10/5:4/337

[3] Interagency Microbiological Risk Assessment Guideline. May 13, 2008, Version 2.6.

[4] Taylor et al, Sensitivity Analysis, "Worst Case", and Maximum Possible Risk (MPR), Proc, $11^{\text {th }}$ Joint Conf on Information Sciences, 2008

[5] Nichols, Tonya, Introduction and Overall Challenges to Microbial Risk Assessment, The Joint U.S. Environmental Protection Agency and Dept of Homeland Security Conference on Real-World Applications and Solutions for Microbial Risk Assessment, April 8-10, 2008

[6] Zadeh, L. "Fuzzy Sets as a Basis for a Theory of Possibility," Fuzzy Sets and Systems1:1 pp 3-28, 1978

[7] Whalen et al, Possibilistic Risk and Counterfactual Probabilities, Proc of the $11^{\text {th }}$ Joint Conf, on Information Sciences, 2008

[8] Bian et al, Explicit Spatial-Temporal Simulation of a Rare Disease, Proc, $11^{\text {th }}$ Joint Conf on Information Sciences, 2008

[9] Schuetz et al, Maximum Possible Risk Modeling, Proc, $11^{\text {th }}$ Joint Conf on Information Sciences, 2008

[10] Gray, George, Overview of Microbial Risk Assessment Activities in EPA's Office of Research and Development, The Joint U.S. Environmental Protection Agency and Dept of Homeland Security Conference on Real-World Applications and Solutions for Microbial Risk Assessment, April 810, 2008 